\title{
POSTOPERATIVE PATIENT CONTROLLED EPIDURAL OR INTRAVENOUS PAIN TREATMENTAFTER ONE STAGE UNILATERAL HYBRID ATRIAL FIBRILLATION ABLATION?
}

Vincent Umbrain MD PhD ${ }^{\alpha}$, Stefan Beckers MD, Gian Battista Chierchia MD PhD ${ }^{\beta}$, Carlo De Asmundis MD PhD ${ }^{\beta}$, Elisabeth De Waele MD PhD r, Marc Diltoer MD PhD r, Mark La Meir MD PhD $\delta$

From the Departments of Anesthesiology and Perioperative Pain ${ }^{\alpha}$, Heart Rhythm Management Centre ${ }^{\beta}$, Intensive care ${ }^{\gamma}$ and, , and Cardiac Surgery ${ }^{\delta}$ of the Universitair Ziekenhuis Brussel, Vrije Universiteit Brussel, Laarbeeklaan 1011090 Brussels, Belgium.

\section{Introduction}

The postoperative pain treatment after one-stage hybrid surgery for atrial fibrillation (AF) ablation presents interesting challenges for the pain physician as several nerve endings of the pleural cavity, the heart, the oesophagus and the throat are sensitised during surgery which in turn may trigger postoperative pain. ${ }^{1}$

\section{Methods}

This retrospective pain study included, after prior approval by the ethical Committee (BUN 143201213077), 69 consecutive patients scheduled for unilateral, one-stage hybrid radiofrequency ablation of atrial fibrillation. The surgical procedure has been described ${ }^{2}$ and was performed under general anaesthesia with an endobronchial blocker for selective lung ventilation. Anaesthesia was induced with sufentanil (0.1-0.2 $\mu \mathrm{g} / \mathrm{kg}$ ), propofol $1-2 \mathrm{mg} / \mathrm{g}$ and vecuronium $0.6 \mathrm{mg} / \mathrm{kg}$ and maintained with inhalation sevoflurane anesthesia ([0-3\%]), sufentanil (range [20-100 $\mu \mathrm{g}$ ] and rocuronium supplements. The patient' options to treat postoperative pain were either a thoracic epidural with ropivacaine (EA) or an i.v. piritramidedehybrobenzperidol pump (IA).

The initial T4-epidural regimen - consisting of a ropivacaine $2 \mathrm{mg} / \mathrm{ml}$ solution - was started at $4 \mathrm{ml} / \mathrm{h}$ during thoracoscopic skin closure. Patient-controlled top-ups in case of residual pain, were possible with a $2 \mathrm{ml}$ dose given every $30 \mathrm{~min}$. Patients receiving the patient controlled intravenous analgesia regimen (IA) had a pump set initially at $2 \mathrm{mg}$ bolus/7min, $30 \mathrm{mg} / 4 \mathrm{~h}$ limit. Before skin closure, all patients were given $20 \mathrm{ml}$ of ropivacaine $7,5 \mathrm{mg} / \mathrm{ml}$ wound infiltration of their intercostal thoracoscopic wounds. Moreover, all patients received paracetamol $1 \mathrm{~g} /$ (Q6h) with a starting dose $1 \mathrm{~h}$ before induction. Postoperatively, in cases of suspicion of pericarditis i.v. diclofenac $75 \mathrm{mg}$ (when possible) or aspirin $500 \mathrm{mg} \mathrm{mg}$ supplemented later with oral $1 \mathrm{mg}$ colchicine and morphine were given. In cases of intractable postoperative pain, a left paravertebral block was provided as a rescue.

Study measurements tools included: for pain intensity assessment, visual analog scale (VAS) scores $(0=$ no pain, 10 worst pain ever) at rest or while coughing and moving at $2 \mathrm{~h}$ and at $6 \mathrm{~h}$ after surgery or more when judged necessary. After day 2, VAS scores were taken twice daily unless considered insufficient. Maximal dynamic VAS scores were also recorded. The localization of pain was specifically asked for. Patients could point out several areas of pain. The pain type was assessed as continuous or intermittent, breathing- or movement-related. Possible side effects of pain treatment such as nausea, vomiting, sedation, respiratory depression, urinary retention, hypotension, epidural catheter kinking, disconnection, pump failures were recorded. Hourly RR and 6 h-evaluation of breathing mode were also evaluated. The intensive care physician had 3 options of for breathing mode assessment: superficial, tense or normal. ABG gases were taken 2 and $6 \mathrm{~h}$ after ICU arrival or when deemed necessary. Sleep duration before surgery and the cumulative sleep hours the first night after surgery (taking the average from the ICU nurses' estimation and the patient's estimation) was recorded. After his first night in ICU the sleep quality score was asked to the patient with two options: good or bad night. Statistical analyses were conducted using SPSS Statistics using when appropriate Mann Whitney $U$ test or Chi-squared analysis with Yates correction.

\section{Results}

Fifty patients were finally included (25/group), Demographic variables and medication were comparable in both groups

$$
\text { EA IA }
$$

$\begin{array}{lcl}\begin{array}{l}\text { Pain-related parameters } \\ \text { Maximal Dynamic VAS }\end{array} & 40 \pm 18 & 45 \pm 20 \\ \begin{array}{c}\text { Pain localization (48h) } \\ \text { Thoracic }\end{array} & 13 / 25 & 19 / 25 \\ \text { Precordial } & 15 / 25 & 13 / 25 \\ \text { Back (mid) } & 16 / 25 & 12 / 25 \\ \text { Epigastric } & 2 / 25 & 1 / 25 \\ \text { Shoulder } & 8 / 25 & 8 / 25 \\ \text { Neck } & 5 / 25 & 4 / 25 \\ \text { Throat } & 1 / 25 & 2 / 25 \\ \text { Pain type } & & \\ \text { Intermittent } & 12 / 25 & 6 / 25 \\ \text { Continuous } & 5 / 25 & 19 / 25^{\star} \\ \text { Movement-related } & 22 / 25 & 23 / 25 \\ \text { Breathing related } & 5 / 25 & \mathbf{2 2 / 2 5} \\ \text { Duration IA/ EA (d) } & 2.6 \pm 0.8 & 1,8 \pm 0.9 \\ \text { Other analgesic agents } & & \\ \text { Paracetamol } & 25 / 25 & 25 / 25 \\ \text { NSAID } & 4 / 25 & 1 / 25 \\ \text { Morphine } & 18 / 25 & 20 / 25 \\ \text { Colchicine } & 9 / 25 & 16 / 25 \\ \text { Aspirin } & 11 / 25 & 19 / 25\end{array}$

\section{Breathing related parameters}

Max breathing rate $1^{\text {st }} 24 \mathrm{~h}: \quad 24 \pm 6$

Breathing pattern:

$\begin{array}{lll}\text { Shallow, superficial } & 8 / 25 & \mathbf{1 5} / 25^{\star} \\ \text { Tense } & 5 / 25 & \mathbf{1 2 / 2 5 ^ { * }} \\ \text { Normal } & 12 / 25 & 4 / 25 \\ \mathrm{O}_{2} \text { levels }(\mathrm{mm} \mathrm{Hg}) & 42 \pm 5 & \mathbf{4 5 \pm 4 ^ { * }}\end{array}$

Max art $\mathrm{CO}_{2}$ levels $(\mathrm{mm} \mathrm{Hg}) \quad 42 \pm 5 \quad \mathbf{4 5 \pm 4 ^ { * }}$

Removal of thorax drain (d) $\quad 1.1 \pm 0.3 \quad 1.3 \pm 0.5$

\section{Conclusion}

$$
\left({ }^{*}=p<0,05\right)
$$

We observed no differences in pain report or length of stay when comparing systemic piritramide to thoracic epidural ropivacaine for patients undergoing a unilateral one stage hybrid atrial fibrillation ablation surgery. An improved recuperation profile with lesser respiratory complaints with a feeling of better nighttime rest during the first $24 \mathrm{~h}$, and a tendency of less postoperative nausea or emesis incidence in the first postoperative days was observed after thoracic epidural ropivacaine administration.

These pros should however be balanced against the potential risk of epidural catheterization. Pericarditis- related pain complaints after surgery are an important issue after unilateral one -stage hybrid AF ablation surgery and equally present after local anaesthetic epidural or systemic opioid patient-controlled approaches.

\section{References}

1. Umbrain et al: Anaesthetic considerations for hybrid atrial fibrillation surgery. Br J Anaesth. 2017; 118(5):798-799 2. Umbrain V et al: One-stage Approach for Hybrid Atrial Fibrillation Treatment. Arrhythmia \& Electrophysiology Review 2017; 6:210-6 